PROCEEDINGS OF THE

AMERICAN MATHEMATICAL SOCIETY

Volume 131, Number 2, Pages 401-408

S 0002-9939(02)06531-0

Article electronically published on September 19, 2002

\title{
HARMONIC BERGMAN FUNCTIONS AS RADIAL DERIVATIVES OF BERGMAN FUNCTIONS
}

\author{
BOO RIM CHOE, HYUNGWOON KOO, AND HEUNGSU YI
}

(Communicated by Juha M. Heinonen)

\begin{abstract}
In the setting of the half-space of the euclidean $n$-space, we show that every harmonic Bergman function is the radial derivative of a Bergman function with an appropriate norm bound.
\end{abstract}

\section{INTRODUCTION}

For a positive integer $n \geq 2$, let $\mathbf{H}=\mathbf{R}^{n-1} \times \mathbf{R}_{+}$denote the upper half $n$-space where $\mathbf{R}_{+}$is the set of all positive real numbers. We will often write a point $z \in \mathbf{H}$ as $z=\left(z^{\prime}, z_{n}\right)$ where $z^{\prime}=\left(z_{1}, \ldots, z_{n-1}\right) \in \mathbf{R}^{n-1}$ and $z_{n} \in \mathbf{R}_{+}$.

For $1 \leq p<\infty$, a harmonic function $u$ on $\mathbf{H}$ is called an $L^{p}$-Bergman function if

$$
\|u\|_{p}=\left\{\int_{\mathbf{H}}|u(z)|^{p} d z\right\}^{1 / p}<\infty .
$$

We let $b^{p}$ denote the space of all $L^{p}$-Bergman functions on $\mathbf{H}$. The space $b^{p}$ is a closed subspace of $L^{p}=L^{p}(\mathbf{H})$ and hence a Banach space.

A harmonic function $u$ on $\mathbf{H}$ is called a Bloch function if

$$
\|u\|_{\mathcal{B}}=\sup z_{n}|\nabla u(z)|<\infty,
$$

where the supremum is taken over all $z \in \mathbf{H}$ and $\nabla$ denotes the gradient operator. We let $\mathcal{B}$ denote the space of all Bloch functions on $\mathbf{H}$. In [5], it is shown that the dual space of $b^{1}$ can be identified with $\mathcal{B} / \mathbf{C}$. So we can consider the harmonic Bloch space $\mathcal{B}$ as a limiting space of the harmonic Bergman space $b^{p}$.

It is elementary to see that, given $a \in \mathbf{H}$ and a harmonic function $u$ on $\mathbf{H}$, there corresponds a unique harmonic function $f$ on $\mathbf{H}$ such that $f(a)=0$ and

$$
u(z)-u(a)=\sum_{j=1}^{n}\left(z_{j}-a_{j}\right) D_{j} f(z), \quad z \in \mathbf{H} .
$$

Here and elsewhere, $D_{j}$ denotes the differentiation with respect to the $j$-th variable for each $j$. In [3] the authors have shown that the maps $u \mapsto D_{j} f$ are bounded on $b^{p}$ if and only if $p>n$, while the map $u \mapsto f$ is not bounded on $b^{p}$ for every $p \geq 1$. Also, these properties are shown to extend to the space $\mathcal{B}$. Note that $b^{p}$-functions

Received by the editors April 18, 2001.

2000 Mathematics Subject Classification. Primary 31B05, 31B10; Secondary 32A36.

Key words and phrases. Bergman functions, radial derivative, upper half-space.

This study was supported in part by the Research Grant of Kwangwoon University in 2001, KOSEF 2000-1-10100-001-3 and KOSEF 98-0701-03-01-5. 
must vanish at $\infty$ along any ray (emanating from the origin). Motivated by these observations, we asked ourselves what would happen if the reference point $a \in \mathbf{H}$ would be replaced by the boundary point $\infty$ and found situations quite different, which is the main result of this paper.

In the following, we let $\mathcal{R}$ denote the radial differentiation of $h \in C^{1}(\mathbf{H})$ defined by

$$
\mathcal{R} h(z)=\sum_{j=1}^{n} z_{j} D_{j} h(z), \quad z \in \mathbf{H} .
$$

Note that $\mathcal{R} h \equiv 0$ if and only if $h$ is radially constant, i.e., $h(z)=h(t z)$ for all $t>0$ and $z \in \mathbf{H}$. The following is our main result.

Theorem 1.1. Let $1 \leq p<\infty$. Then, given $u \in b^{p}$, there corresponds a unique $\widetilde{u} \in b^{p}$ such that $u=\mathcal{R} \widetilde{u}$. In addition, we have the following:

(1) The map $u \mapsto \widetilde{u}$ is bounded on $b^{p}$.

(2) For $1 \leq p<n$, there exists a positive constant $C_{p}$ such that

$$
\sum_{j=1}^{n}\left\|D_{j} \widetilde{u}\right\|_{p} \leq C_{p}\left\|D_{n} u\right\|_{p}
$$

for all $u \in b^{p}$.

Remarks. (1) For the representation $u=\mathcal{R} \widetilde{u}$, we will define $\widetilde{u}$ as an integral involving $u$ as follows:

$$
\widetilde{u}(z)=-\int_{1}^{\infty} \frac{u(t z)}{t} d t .
$$

The key of this integral representation is that $b^{p}$-functions vanish with suitable order at $\infty$ along the ray passing through $z$. However, this vanishing property is not shared by Bloch functions, which might have relatively wild (actually logarithmic 1]) behavior near $\infty$. Thus, one may not expect such a representation for Bloch functions, as turns out to be the case. See Proposition 3.1.

(2) Note that the partial derivatives $D_{j} \widetilde{u}$ are obtained by differentiating an integral of $u$. Thus, one may roughly expect that the functions $D_{j} \widetilde{u}$ and $u$ are approximately of the same growth. We show that this is not the case in the sense that one cannot replace $D_{n} u$ by $u$ in the right side of (1.1). See Proposition 3.2.

(3) The inequality (1.1) does not mean $\left\|D_{n} u\right\|_{p}<\infty$ at all. In fact, if $\left\|D_{n} u\right\|_{p}<$ $\infty$ would happen for functions $u \in b^{p}$, then all the partial derivatives of $b^{p}$-functions would also be $b^{p}$-functions by Lemma 2.2 below, which cannot be expected in general. An explicit proof showing the existence of $u \in b^{p}$ with $D_{n} u \notin b^{p}, 1 \leq p<$ $\infty$, is included for completeness. See Proposition 3.3 Also, for $1 \leq p<\infty$, we have

$$
\left\|z_{n} D_{n} u\right\|_{p} \approx\|u\|_{p}
$$

as $u$ ranges over all $b^{p}$-functions. See [5].

(4) The range of $p$ for the inequality (1.1) is sharp. That is, (1.1) fails to hold for $n \leq p<\infty$. See Proposition 3.4

Section 2 is devoted to the proof of Theorem 1.1 In Section 3 we provide examples concerning the above remarks.

Constants. Throughout the paper we will use the same letter $C$ to denote various constants, often with subscripts indicating dependency, which may change at each occurrence. We will often write $A \lesssim B$ or $B \gtrsim A$ for nonnegative quantities $A, B$ 
if $A$ is dominated by $B$ times some inessential positive constant. Also, we write $A \approx B$ if $A \lesssim B$ and $A \gtrsim B$.

\section{Proof of Theorem 1.1}

Before proceeding to the proof, we review some preliminary results on reproducing kernels for the spaces $b^{p}$. The reproducing kernel $R(z, w)$ for $b^{p}$ is given by

$$
R(z, w)=\frac{4}{n \sigma_{n}} \cdot \frac{n\left(z_{n}+w_{n}\right)^{2}-|z-\bar{w}|^{2}}{|z-\bar{w}|^{n+2}}, \quad z, w \in \mathbf{H},
$$

where $\sigma_{n}$ denotes the volume of the unit ball in $\mathbf{R}^{n}$ and $\bar{w}=\left(w^{\prime},-w_{n}\right)$. That is, we have

$$
u(z)=\int_{\mathbf{H}} u(w) R(z, w) d w, \quad z \in \mathbf{H},
$$

for all $u \in b^{p}, 1 \leq p<\infty$. See [2], [5] for details and related topics. A generalized reproducing property of the kernel $R(z, w)$ is also available [5]:

$$
u(z)=-2 \int_{\mathbf{H}} w_{n}\left[D_{n} u(w)\right] R(z, w) d w, \quad z \in \mathbf{H},
$$

for all $u \in b^{p}, 1 \leq p<\infty$. This generalized reproducing formula shows that $b^{p}$ functions are completely determined by their normal derivatives. In particular, it is not too surprising to see that the $L^{p}$-size of derivatives of $b^{p}$-functions is controlled by that of normal derivatives. To see this, we first need a lemma, which is a special case of Lemma 4.4 of [4].

Lemma 2.1. Let $1 \leq p<\infty$. For $\psi \in L^{p}$, define

$$
T \psi(z)=\int_{\mathbf{H}} \psi(w) \frac{w_{n}}{|z-\bar{w}|^{n+1}} d w, \quad z \in \mathbf{H} .
$$

Then, $T: L^{p} \rightarrow L^{p}$ is bounded.

Lemma 2.2. Let $1 \leq p<\infty$. Then we have

$$
\sum_{j=1}^{n}\left\|D_{j} u\right\|_{p} \leq C_{p}\left\|D_{n} u\right\|_{p}
$$

for functions $u \in b^{p}$.

Proof. By a straightforward calculation using (2.1), it is easily checked that

$$
\left|D_{j} R(z, w)\right| \lesssim \frac{1}{|z-\bar{w}|^{n+1}}, \quad z, w \in \mathbf{H},
$$

for all $j$. Here, the ambiguous notation $D_{j} R(z, w)$ means $D_{j}[R(\cdot, w)]$ evaluated at $z$ for each fixed $w$. Thus, by (2.2) and (2.3), we obtain

$$
\left|D_{j} u(z)\right| \lesssim \int_{\mathbf{H}}\left|D_{n} u(w)\right| \frac{w_{n}}{|z-\bar{w}|^{n+1}} d w, \quad z \in \mathbf{H},
$$

for each $j$. Now, the lemma follows from Lemma 2.1. The proof is complete. 
We are now ready to prove Theorem 1.1

Proof of Theorem 1.1. Since any $b^{p}$-function vanishes at $\infty$ along any ray, the uniqueness follows from the fact that radially constant functions vanishing at $\infty$ along any ray must be identically 0 . Let $1 \leq p<\infty$, fix $u \in b^{p}$ and define

$$
\widetilde{u}(z)=-\int_{1}^{\infty} \frac{u(t z)}{t} d t, \quad z \in \mathbf{H} .
$$

By the mean-value property, Jensen's inequality and Cauchy's estimates, we have (see Corollary 8.2 of [2])

$$
\left|D^{\alpha} u(t z)\right| \leq C_{\alpha} \frac{\|u\|_{p}}{\left(t z_{n}\right)^{|\alpha|+n / p}}, \quad t>0, \quad z \in \mathbf{H},
$$

for every multi-index $\alpha$. It follows that

$$
\int_{1}^{\infty} \frac{\left|D^{\alpha} u(t z)\right|}{t} d t<\infty
$$

for each $z \in \mathbf{H}$ and multi-index $\alpha$. Taking $\alpha=(0,0, \ldots, 0)$, we see that $\widetilde{u}$ is well defined. Also, via the dominated convergence theorem, we see that differentiation, as many times as we want, under the integral sign of (2.4), is justified. In particular, $\widetilde{u}$ is harmonic on $\mathbf{H}$ and for each $j$ we have

$$
D_{j} \widetilde{u}(z)=-\int_{1}^{\infty} D_{j} u(t z) d t .
$$

Now, since $u$ vanishes at $\infty$ along any ray, we have by (2.5)

$$
\begin{aligned}
u(z) & =-\int_{1}^{\infty} \frac{d u}{d t}(t z) d t \\
& =-\int_{1}^{\infty} \sum_{j=1}^{n} z_{j} D_{j} u(t z) d t \\
& =-\sum_{j=1}^{n} z_{j} \int_{1}^{\infty} D_{j} u(t z) d t \\
& =\sum_{j=1}^{n} z_{j} D_{j} \widetilde{u}(z) \\
& =\mathcal{R} \widetilde{u}(z)
\end{aligned}
$$

for all $z \in \mathbf{H}$. This proves the existence.

We now prove (1) and (2). First, by Minkowski's integral inequality we have

$$
\begin{aligned}
\|\widetilde{u}\|_{p} & \leq \int_{1}^{\infty}\left\{\int_{\mathbf{H}}|u(t z)|^{p} d z\right\}^{1 / p} \frac{d t}{t} \\
& =\int_{1}^{\infty}\left\{\int_{\mathbf{H}}|u(z)|^{p} d z\right\}^{1 / p} \frac{d t}{t^{1+n / p}} \\
& =\|u\|_{p} \int_{1}^{\infty} \frac{d t}{t^{1+n / p}} .
\end{aligned}
$$


Since the above integral is finite for all $1 \leq p<\infty$, we have (1). Next, by Minkowski's integral inequality again we have

$$
\begin{aligned}
\left\|D_{j} \widetilde{u}\right\|_{p} & \leq \int_{1}^{\infty}\left\{\int_{\mathbf{H}}\left|D_{j} u(t z)\right|^{p} d z\right\}^{1 / p} d t \\
& =\int_{1}^{\infty}\left\{\int_{\mathbf{H}}\left|D_{j} u(z)\right|^{p} d z\right\}^{1 / p} \frac{d t}{t^{n / p}} \\
& =\left\|D_{j} u\right\|_{p} \int_{1}^{\infty} \frac{d t}{t^{n / p}}
\end{aligned}
$$

for each $j$. Note that the above integral is finite for $1 \leq p<n$. This, together with Lemma 2.2, yields (2). The proof is complete.

\section{EXAMPLES}

In this section we provide examples concerning the remarks in the Introduction. First, we show that the radial derivative representation fails to hold in general for Bloch functions.

Proposition 3.1. There is a function $u \in \mathcal{B}$ such that $u \neq \mathcal{R} v$ for any $v \in \mathcal{B}$.

Proof. Let $u(z)=\log \left(z_{1}^{2}+z_{n}^{2}\right)$ for $z \in \mathbf{H}$. Then we can easily check that $u \in \mathcal{B}$. Suppose $u=\mathcal{R} v$ for some $v \in \mathcal{B}$. Then we have

$$
\log \left(z_{1}^{2}+z_{n}^{2}\right)=\sum_{j=1}^{n} z_{j} D_{j} v(z)
$$

for all $z \in \mathbf{H}$. By plugging $(0, \ldots, 0, m)$ into $z$ and then letting $m \rightarrow \infty$ on both sides of (3.1), we see that the left side of (3.1) is unbounded but the right side of (3.1) is bounded by $\|v\|_{\mathcal{B}}$. Therefore we get a contradiction and the proof is complete.

Next, we show that (1.1) is no longer true if $\left\|D_{n} u\right\|_{p}$ is replaced by $\|u\|_{p}$. For that purpose, it suffices to show that the map $u \mapsto D_{j} \widetilde{u}$ cannot be bounded on any $b^{p}, 1 \leq p<\infty$.

Proposition 3.2. Given $1 \leq p<\infty$, there is a function $u \in b^{p}$ satisfying $D_{n} \widetilde{u} \notin b^{p}$.

Proof. In order to derive a contradiction, suppose that there is some $1 \leq p<\infty$ for which $D_{n} \widetilde{u} \in b^{p}$ for all $u \in b^{p}$. Recall that the map $u \mapsto \widetilde{u}$ is bounded on $b^{p}$ by Theorem 1.1 Thus, since the convergence in $b^{p}$ implies uniform convergence on compact subsets, the closed graph theorem implies that the map $u \mapsto D_{n} \widetilde{u}$ is bounded on $b^{p}$.

Now, let

$$
\varphi(z)= \begin{cases}\log |z| & \text { for } n=2, \\ |z|^{2-n} & \text { for } n \geq 3\end{cases}
$$

and let $v(z)=D_{n}^{3} \varphi(z)$ for $z \in \mathbf{H}$. Then it is easy to see that

$$
v(z)=\frac{f(z)}{|z|^{n+4}}, \quad D_{n} v(z)=\frac{g(z)}{|z|^{n+6}}
$$


for some homogeneous harmonic polynomials $f$ and $g$ of degree 3 and 4 , respectively. For $\delta>0$, put $v_{\delta}(z)=v\left(z^{\prime}, z_{n}+\delta\right), z \in \mathbf{H}$. Then, by homogeneity of $f$, we have

$$
\begin{aligned}
\left\|v_{\delta}\right\|_{p}^{p} & =\int_{\mathbf{H}} \frac{\left|f\left(z^{\prime}, z_{n}+\delta\right)\right|^{p}}{\left|\left(z^{\prime}, z_{n}+\delta\right)\right|^{p(n+4)}} d z \\
& =\frac{\delta^{n+3 p}}{\delta^{p(n+4)}} \int_{\mathbf{H}} \frac{\left|f\left(z^{\prime}, z_{n}+1\right)\right|^{p}}{\left|\left(z^{\prime}, z_{n}+1\right)\right|^{p(n+4)}} d z \\
& \approx \delta^{n-p n-p} \int_{\mathbf{H}} \frac{|f(z)|^{p}}{1+|z|^{p(n+4)}} d z \\
& \lesssim \delta^{n-p n-p}\left(\sup _{|\zeta|=1}|f(\zeta)|^{p}\right) \int_{\mathbf{H}} \frac{d z}{1+|z|^{p(n+1)}} .
\end{aligned}
$$

Since the last integral of the above is finite, we see $v_{\delta} \in b^{p}$ and

$$
\left\|v_{\delta}\right\|_{p} \approx \delta^{n / p-n-1} .
$$

On the other hand, by (2.5) and homogeneity of $g$, we have

$$
\begin{aligned}
\left\|D_{n} \widetilde{v}_{\delta}\right\|_{p}^{p} & =\int_{\mathbf{H}}\left|\int_{1}^{\infty} \frac{g\left(t z^{\prime}, t z_{n}+\delta\right)}{\left|\left(t z^{\prime}, t z_{n}+\delta\right)\right|^{n+6}} d t\right|^{p} d z \\
& =\frac{\delta^{n+4 p}}{\delta^{p(n+6)}} \int_{\mathbf{H}}\left|\int_{1}^{\infty} \frac{g\left(t z^{\prime}, t z_{n}+1\right)}{\left|\left(t z^{\prime}, t z_{n}+1\right)\right|^{n+6}} d t\right|^{p} d z .
\end{aligned}
$$

Note that the last integral above is independent of $\delta$. If it is not finite (actually this is the case for $n \leq p<\infty)$, then we already have a contradiction. If it is finite (actually this is the case for $p<n$ ), then we have

$$
\left\|D_{n} \widetilde{v}_{\delta}\right\|_{p} \approx \delta^{n / p-n-2}
$$

and thus by (3.2)

$$
\frac{\left\|D_{n} \widetilde{v}_{\delta}\right\|_{p}}{\left\|v_{\delta}\right\|_{p}} \approx \delta^{-1} \rightarrow \infty \quad \text { as } \quad \delta \rightarrow 0 .
$$

This shows that the map $u \mapsto D_{n} \widetilde{u}$ is not bounded on $b^{p}$, which is again a contradiction. The proof is complete.

We also show that the right side of (1.1) is possibly infinite.

Proposition 3.3. Given $1 \leq p<\infty$, there is a function $u \in b^{p}$ satisfying $D_{n} u \notin b^{p}$.

Proof. In order to derive a contradiction, suppose that there is some $1 \leq p<\infty$ for which $D_{n} u \in b^{p}$ for all $u \in b^{p}$. Then, we again see via the closed graph theorem that the map $u \mapsto D_{n} u$ is bounded on $b^{p}$.

We continue using the notations defined in the proof of Proposition 3.2 Using a similar argument as in the estimate of $\left\|v_{\delta}\right\|_{p}$, one can verify that

$$
\left\|D_{n} v_{\delta}\right\|_{p} \approx \delta^{n / p-n-2} \text {. }
$$

This, together with (3.2), yields

$$
\frac{\left\|D_{n} v_{\delta}\right\|_{p}}{\left\|v_{\delta}\right\|_{p}} \approx \delta^{-1} \rightarrow \infty \quad \text { as } \quad \delta \rightarrow 0
$$

This shows that the map $u \mapsto D_{n} u$ is not bounded on $b^{p}$, which is a contradiction as desired. The proof is complete. 
Finally, we show that (1.1) is sharp for the range of $p$. For that purpose, it suffices to show the following.

Proposition 3.4. There is a function $u \in \bigcap_{p \geq n} b^{p}$ satisfying $D_{n} u \in \bigcap_{p \geq n} b^{p}$, but $D_{n} \widetilde{u} \notin \bigcup_{p \geq n} b^{p}$.

Proof. First, consider the case $n>2$. Fix $z_{0}=\left(0^{\prime}, 1\right)$. Let $\varphi(z)=\left|z+z_{0}\right|^{2-n}$ and put $u=c_{n} D_{1} D_{2} \cdots D_{n-1} \varphi$ where $c_{n}$ is chosen so that

$$
u(z)=\frac{z_{1} z_{2} \cdots z_{n-1}}{\left|z+z_{0}\right|^{3 n-4}}, \quad z \in \mathbf{H} .
$$

Harmonicity of $\varphi$ on $\mathbf{H}$ implies that of $u$. A straightforward calculation yields

$$
D_{n} u(z)=(4-3 n) \frac{z_{1} z_{2} \cdots z_{n-1}\left(z_{n}+1\right)}{\left|z+z_{0}\right|^{3 n-2}}, \quad z \in \mathbf{H} .
$$

From (3.3) and (3.4), we have

$$
|u(z)| \lesssim(1+|z|)^{3-2 n}, \quad\left|D_{n} u(z)\right| \lesssim(1+|z|)^{2-2 n}
$$

and therefore both $u$ and $D_{n} u$ belong to $b^{p}$ for any $p \geq n$. Also, we have

$$
\begin{aligned}
D_{n} \widetilde{u}(z) & =-\int_{1}^{\infty} D_{n} u(t z) d t \\
& =(3 n-4) z_{1} \cdots z_{n-1} \int_{1}^{\infty} \frac{t^{n-1}\left(t z_{n}+1\right)}{\left|t z+z_{0}\right|^{3 n-2}} d t
\end{aligned}
$$

and therefore

$$
\left|D_{n} \widetilde{u}(z)\right| \gtrsim\left|z_{1} z_{2} \cdots z_{n-1}\right| \int_{1}^{1 /|z|} t^{n-1} d t \approx \frac{\left|z_{1} z_{2} \cdots z_{n-1}\right|}{|z|^{n}}, \quad z \in \mathbf{H} .
$$

Let $E$ be the set of all points $z \in \mathbf{H}$ such that $0<z_{n}<1$ and $z_{n} / 2 \leq z_{j} \leq 2 z_{n}$ for each $j$. Note that $z_{j} \approx z_{n} \approx|z|$ for $z \in E$. Thus, for any $p \geq n$, we have by (3.5)

$$
\begin{aligned}
\int_{\mathbf{H}}\left|D_{n} \widetilde{u}(z)\right|^{p} d z & \geq \int_{E}\left|D_{n} \widetilde{u}(z)\right|^{p} d z \\
& \gtrsim \int_{E}\left\{\frac{z_{1} z_{2} \cdots z_{n-1}}{|z|^{n}}\right\}^{p} d z \\
& \gtrsim \int_{E} \frac{d z}{z_{n}^{p}} \\
& =\left(\frac{3}{2}\right)^{n-1} \int_{0}^{1} \frac{z_{n}^{n-1}}{z_{n}^{p}} d z_{n} \\
& =\infty
\end{aligned}
$$

as desired.

Now, consider the case $n=2$. Let $u$ be the imaginary part of the holomorphic function $(z+i)^{-2}$ on $\mathbf{H}$. Here, $z=(x, y)=x+i y$. Then, we have $D_{2} u(z)=$ $-2 \Re(z+i)^{-3}$. Since

$$
|u(z)| \lesssim(1+|z|)^{-2}, \quad\left|D_{2} u(z)\right| \lesssim(1+|z|)^{-3},
$$


we see that both $u$ and $D_{2} u$ belong to $b^{p}$ for any $p \geq 2$. Note that

$$
\begin{aligned}
D_{2} \widetilde{u}(z) & =-\int_{1}^{\infty} D_{2} u(t z) d t \\
& =2 \Re \int_{1}^{\infty}(t z+i)^{-3} d t \\
& =\Re\left(z^{-1}(z+i)^{-2}\right) \\
& =\frac{x\left(x^{2}-3 y^{2}-4 y-1\right)}{|z|^{2}|z+i|^{4}} .
\end{aligned}
$$

Let $E$ be the set of all points $z \in \mathbf{H}$ such that $|z| \leq 1 / 10$. If $z \in E$, then we have

$$
\left|x^{2}-3 y^{2}-2 y-1\right| \geq 1-\left(x^{2}+3 y^{2}+2 y\right) \geq 1-5|z| \geq \frac{1}{2}
$$

so that

$$
\left|D_{2} \widetilde{u}(z)\right| \gtrsim \frac{|x|}{|z|^{2}}
$$

Therefore, for any $p \geq 2$, we have

$$
\begin{aligned}
\int_{\mathbf{H}}\left|D_{2} \widetilde{u}(z)\right|^{p} d x d y & \geq \int_{E}\left|D_{2} \widetilde{u}(z)\right|^{p} d x d y \\
& \gtrsim \int_{E} \frac{|x|^{p}}{|z|^{2 p}} d x d y \\
& =\int_{0}^{\pi} \int_{0}^{1 / 10} r\left\{\frac{|\cos \theta|}{r}\right\}^{p} d r d \theta \\
& =\infty
\end{aligned}
$$

as desired. This completes the proof.

\section{REFERENCES}

1. H. Ajmi, Harmonic Bloch functions on the upper half-space, Ph.D. Thesis, Michigan State University, 1992.

2. S. Axler, P. Bourdon and W. Ramey, Harmonic function theory, Springer-Verlag, New York, 1992. MR 93f:31001

3. B. R. Choe, H. Koo, and H. Yi, Gleason's problem for harmonic Bergman and Bloch functions on half-spaces, Integr. Equ. Oper. Theory 36 (2000), 269-287. MR 2001c:46040

4. B. R. Choe, and H. Yi, Representations and interpolations of harmonic Bergman functions on half-spaces, Nagoya Math. J. 151 (1998), 51-89. MR 99k:31002

5. W. Ramey and H. Yi, Harmonic Bergman functions on half-spaces, Trans. Amer. Math. Soc. 348(1996), 633-660. MR 96g:31006

Department of Mathematics, Korea University, Seoul 136-701, Korea

E-mail address: choebr@math.korea.ac.kr

Department of Mathematics, Korea University, Seoul 136-701, Korea

E-mail address: koohw@math.korea.ac.kr

Department of Mathematics, Kwangwoon University, Seoul 139-701, Korea

E-mail address: hsyi@gwu.ac.kr 